\title{
SPACE CONTROL IN MIXED DENTITION - SPACE MAINTAINERS
}

\author{
Magda-Mihaela Luca ${ }^{1,2}$, Ștefania Dinu ${ }^{1,2}$, Nicoleta Nikolajevic-Stoican ${ }^{2}$, Simina Boia ${ }^{3}$, Eugen- \\ Radu Boia ${ }^{4}$, Bianca Dragoș ${ }^{5}$, Mălina Popa ${ }^{1,2}$
}

\begin{abstract}
Maintaining the child's oral cavity in optimal health by preserving the morpho-functional integrity of primary teeth, given the influence they have on the growth and development of the stomatognathic system is the duty and responsibility of the pedodontist. In addition to prevention, increased attention should be granted to the space control in case of early loss of primary teeth in the support area, recommending the use of space maintainers until the eruption of permanent successors, to intercept the appearance of future anomalies.
\end{abstract}

Keywords: premature loss, space maintainers, interception

\section{Introduction}

Early loss of primary molars affects millions of patients worldwide with a significant negative impact on somatic growth and development of the stomatognathic system, social function and quality of life. Primary dentition plays an essential role in the healthy growth of the child influencing the development of the orofacial system functions. Exfoliation of primary teeth is a physiological process and is closely related to the development of successive permanent teeth. When this process is interrupted by the premature loss of primary molars due to complications of carious lesions, malocclusions occur resulting in disruption of the integrity of the dental arch that negatively affects the eruption process and proper alignment of permanent teeth [1]. The lack of space through the premature loss of teeth, especially of the primary molars, causes shifting of adjacent teeth into the extraction gap resulting abnormal axial inclination, dental crowding, ectopias, supraalveolodontia of antagonist teeth, mesialized eruption of the first permanent molar. Whenever early loss of primary teeth affects the anterior area there are physiognomic disorders with the appearance of bad oral habits of interposing the tongue or objects [2].

The main factors that lead to the premature loss of primary teeth are trauma and carious lesions complicated with endodontic pathology which often due to the increased permeability of the pulp chamber floor by the presence of furcation canals is usually the cause of furcation lesions, which requires early extraction of these teeth [3].

The best way to avoid these problems is to preserve the primary teeth on the arch until they are exfoliated. There are data in the literature that claim that the primary tooth is the best space maintainer [4]. However, there are currently many children with such problems. Therefore, to prevent these undesired effects, it is mandatory to use space maintainers until the eruption of the permanent successors [5].

The stainless-steel band and loop space maintainers are commonly used in pediatric dentistry, as they are welltolerated by children and the realisation technique is very easy [6].

With the rapid progress of 3D scanning, printing and milling technology in the medical field that has grown in recent times, studies have been performed in which the application of this technology in pediatric dentistry has been attempted in order to obtain aesthetic space maintainers or with better clinical adaptation [7].

\section{Materials and Methods}

Space maintainers are medical devices whose main role is to prevent the loss of space needed for the eruption of permanent teeth, by preventing the mesialization of first permanent molars [8]. These devices can be classified according to several criteria, according to the mode of aggregation they can be fixed, removable and mobile according to their function they can be active or passive.

${ }^{1}$ Department of Pedodontics, Pediatric Dentistry Research Center, Faculty of Dental Medicine, "Victor Babeș" University of Medicine and Pharmacy, Timișoara, Romania

${ }^{2}$ Pediatric Dentistry Research Centre, Pedo-Research, Timişoara, Romania

${ }^{3}$ Department of Periodontology, Faculty of Dental Medicine, "Victor Babeș" University of Medicine and Pharmacy, Timişoara, Romania

${ }^{4}$ Department of ENT, Faculty of Medicine, "Victor Babeș" University of Medicine and Pharmacy, Timișoara, Romania ${ }^{5}$ Phd Student, Department of Propaedeutics and Dental Materials, Faculty of Dental Medicine, "Victor Babeș" University of Medicine and Pharmacy, Timișoara, Romania

E-mail: luca.magda@umft.ro; dinu.stefania@umft.ro; nicoleta.stoican@umft.ro; simina.boia@umft.ro; eugen.boia@umft.ro; bianca.roman@umft.ro; popa.malina@umft.ro 


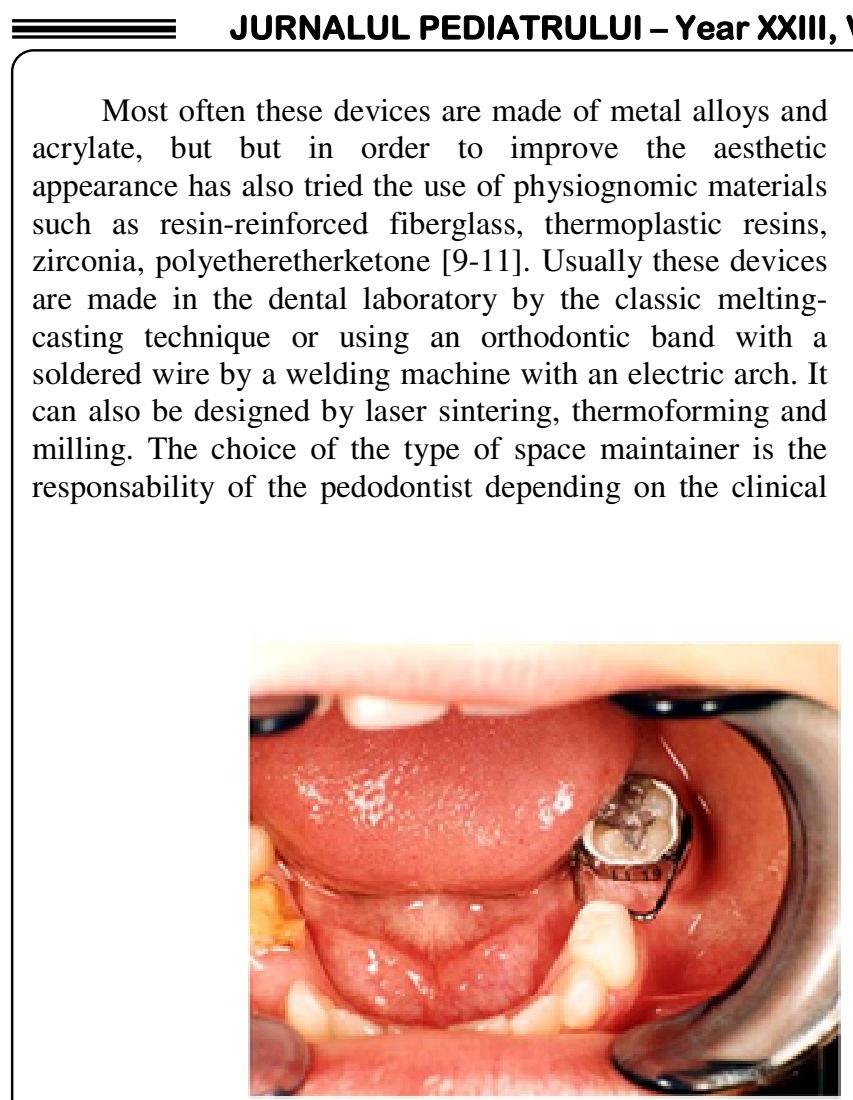

a) situation, which requires to establish an individualized treatment plan.

Types of space maintainers used in the Pedodontics clinic - University of Medicine and Pharmacy Victor BabeșTimișoara [12]:

Case 1 - A 9-year-old patient with early loss of 6.3, $6.4,6.5,7.5,8.4$ following evolutionary decays associated with pulpal complications presented for treatment accusing dental pain. Associated with dental therapy, a first phase was chosen for the application of a passive Band and loop space maintainer made by welding a loop on an orthodontic band cemented with CIS on 3.6 (figure 1).

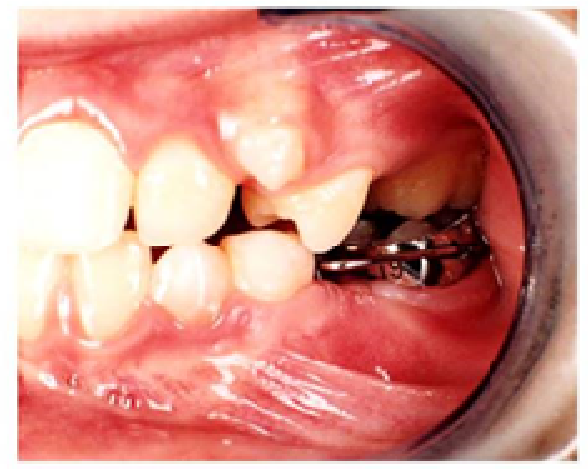

b)

Fig. 1. a) the clinical aspect of the space maintainer; b) the device in occlusion.

Case 2 - 8-year-old patient with early loss of primary molars 6.4, 6.5, following pulpal diseases presented for treatment. Following the analysis of the complementary exams and the exploratory milling, it was found that both
6.4 and 6.5 are no longer restorable. After performing the extractions, it was decided to apply a passive band and loop type space maintainer with long arms welded on an orthodontic band (figure 2).
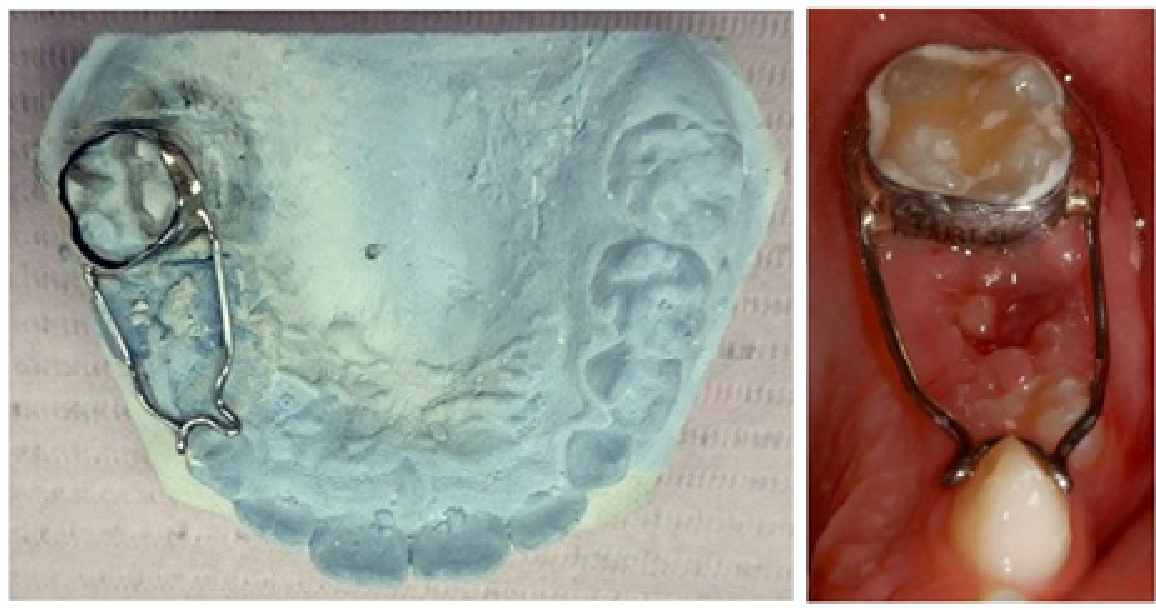

Fig. 2 Band and loop space maintainer - working cast and clinical appearance. 


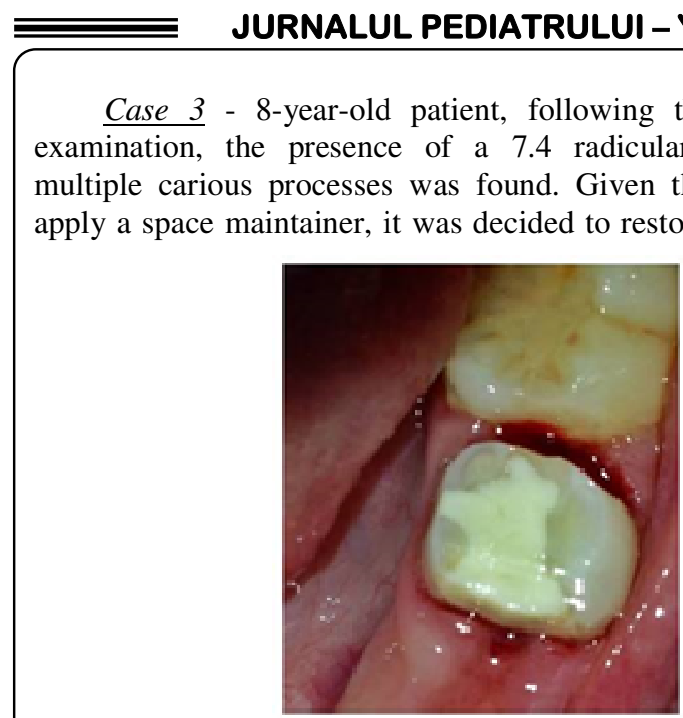

a)

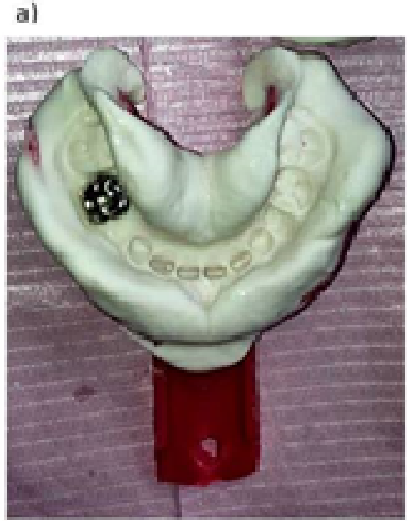

d)

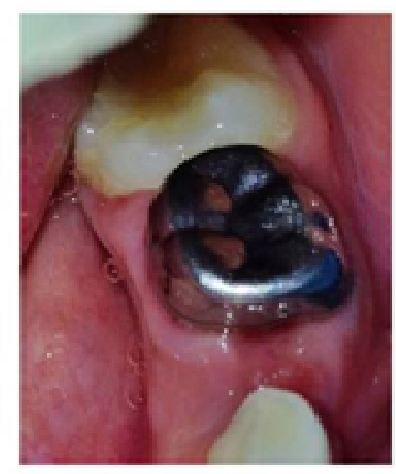

b)

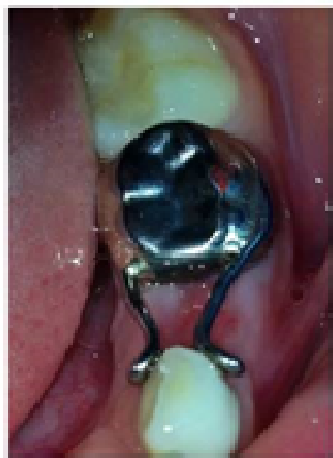

c)

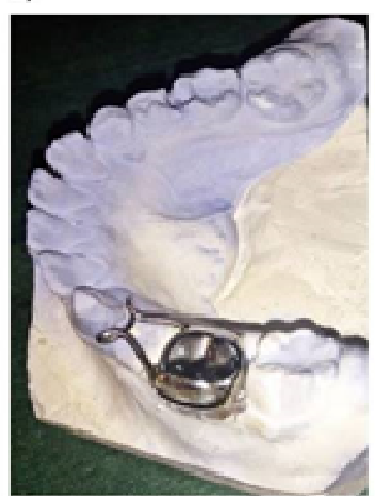

el

Fig. 3. Crown and loop - a) the aspect of the preparation of the primary tooth for the application of the steel pedodontic crown, b) the intraoral aspect of the pedodontic crown, c) the intraoral clinical aspect of the space maintainer, d) the alginate impression for making the space maintainer, e) the aspect on the working cast of the space maintainer.

Case 4 - Patient with mixed dentition, with early bilateral loss of primary molars at the upper arch 5.4, 5.5, 6.4, 6.5 following multiple dental lesions, the patient having an increased cariogenic index. It was chosen for the application of a Nance device (figure 4), it is provided with an acrylic button that fits intimately in the anterior area of the hard palate and a metal component that is welded on orthodontic bands applied to the first permanent upper molars. This device prevents mesial migration of the first permanent molars.

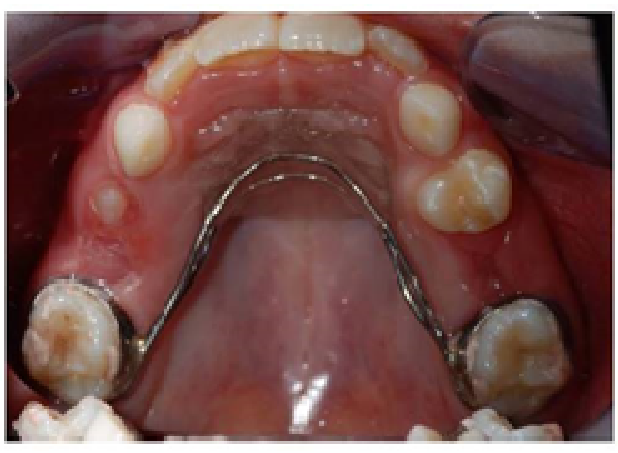

a)

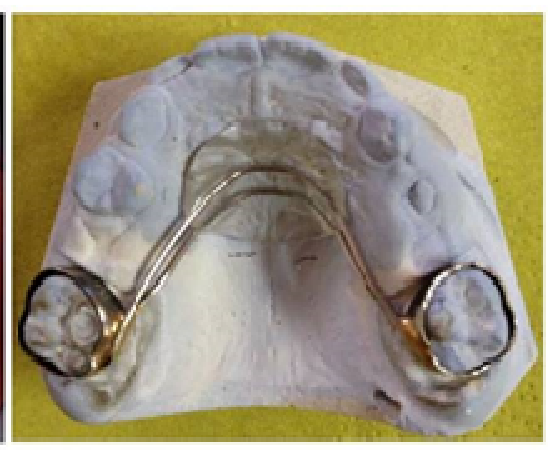

b)

Fig. 4. Nance device: a) intraoral aspect; b) the aspect of the working cast. 


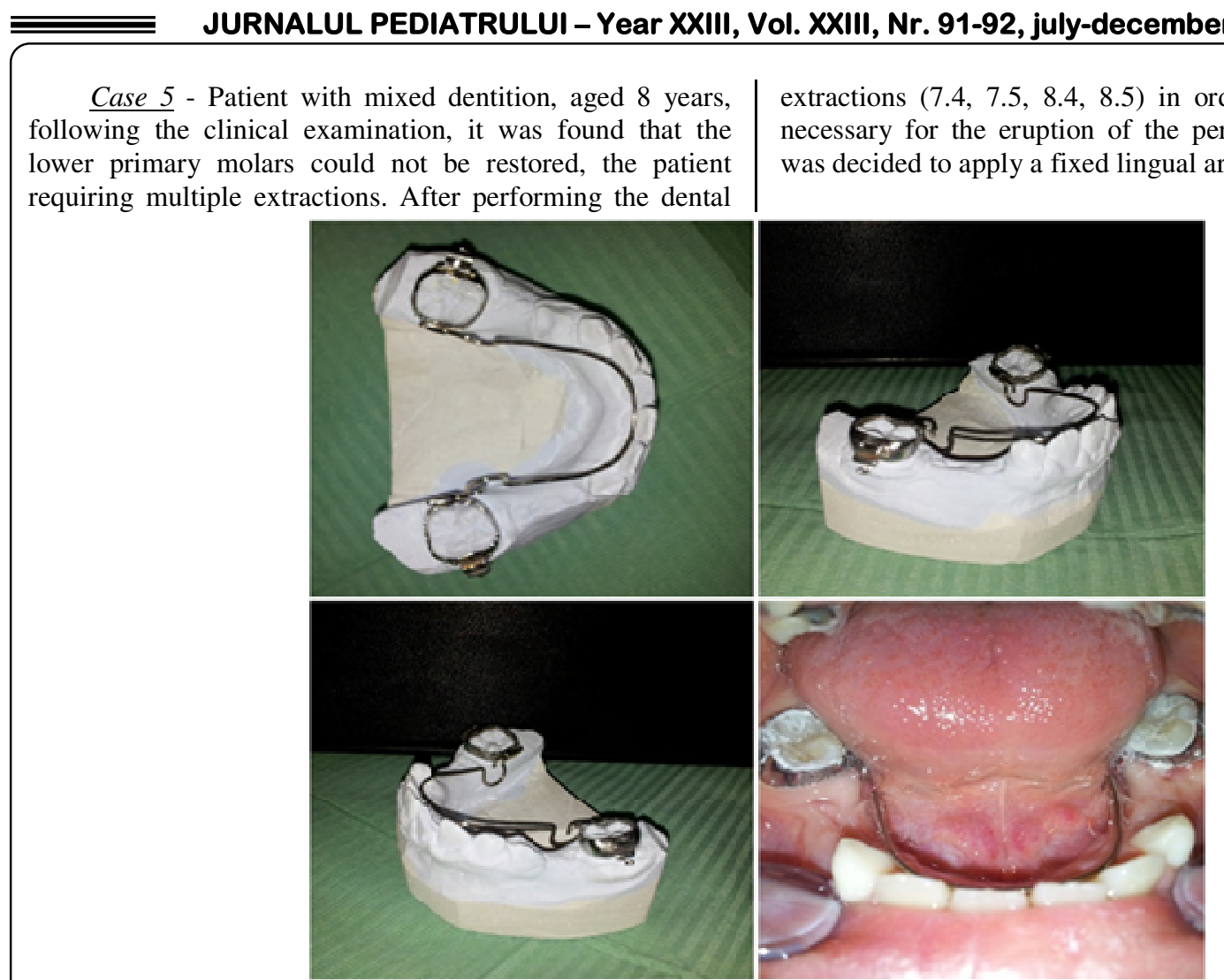

Fig. 5. Lingual arch - intraoral and working cast aspect.

$\underline{\text { Case } 6}$ - Patient with major space loss and dental crowding presented to the clinic for treatment. To correct the compression in the molar region, a removable transpalatal bar (figure 6) was applied, the patient subsequently requiring an orthodontic treatment for the creation of space and dental alignment with the establishment of neutral occlusal ratios.

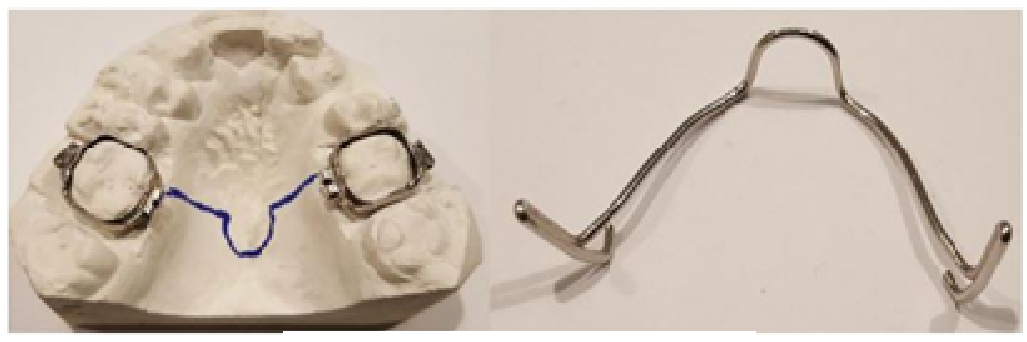

Fig. 6. TPA - removable device.

Case 7 - A 7-year-old patient with early loss of primary molars in the lower arch against the background of carious disease, the patient with a history of bottle caries, presented to the Pedodontic Clinic accusing eruption disorders. A lingual removable space maintainer was applied to the patient, type - lingual appliance (figure 7).
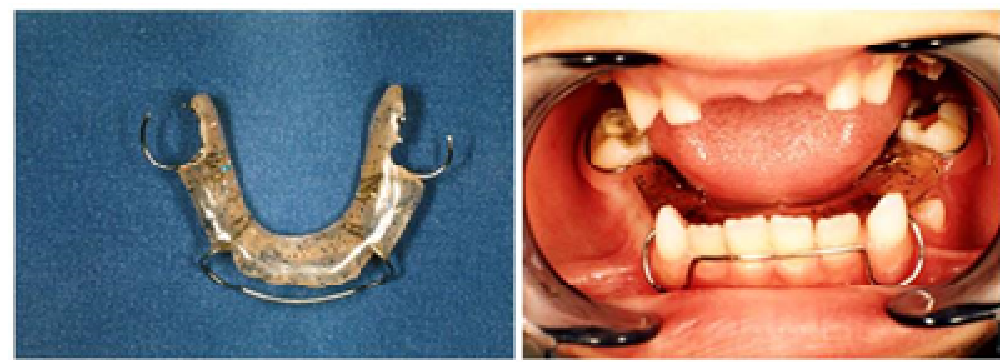

Fig.7. Lingual appliance provided with acrylate space maintainer. 
Case 8 - 8 years-old-pacient, following the clinical examination, it was found the absence from the arch of 7.5 , $7.4,8.4$ following dental extractions performed in the past.
Due to the incomplete eruption of the lower incisor group in order not to interfere with the dental eruption process, it was decided to apply a lingual space maintainer (figure 8).
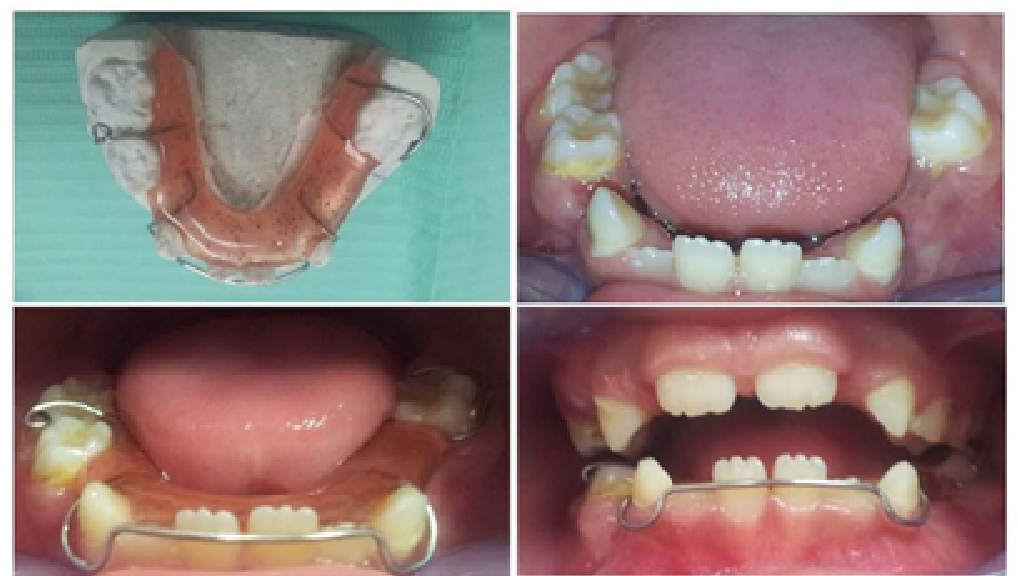

Fig. 8. Lingual appliance provided with anchoring elements, vestibular arch and acrylate space maintainer.

$\underline{\text { Case } 9}$ - 4 year-old patient, with early loss of upper central incisors 5.1, 6.1, following a trauma was rehabilitated by applying a palatal appliance provided with acrylic teeth, artificial gums and hooks in the lateral area for anchoring (figure 9). This therapeutic solution was chosen to restore both the aesthetic function and to improve the phonation.
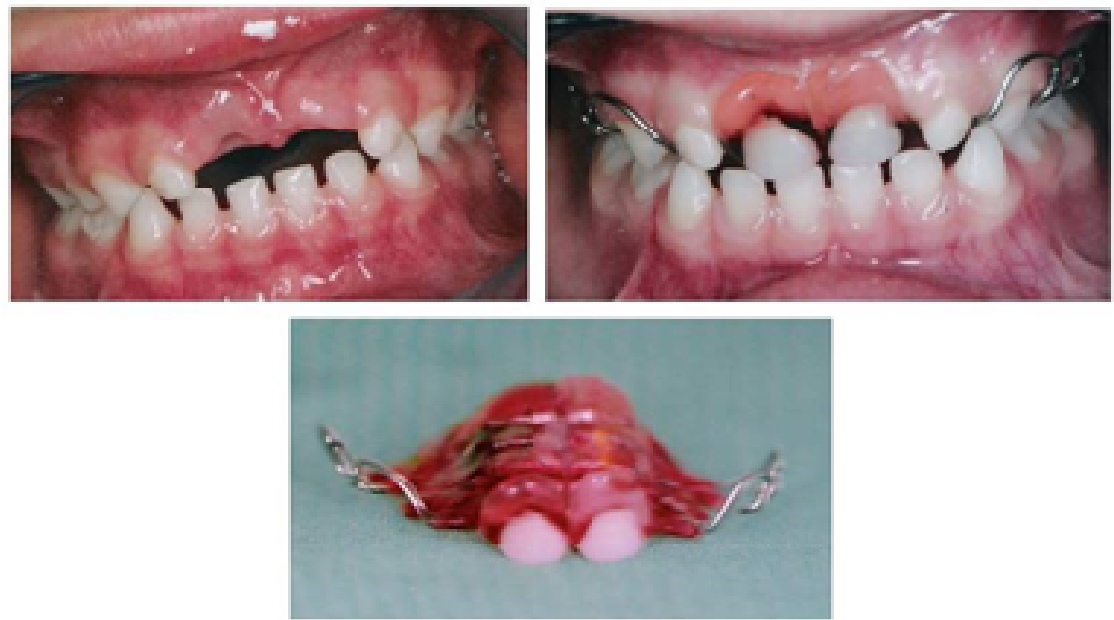

Fig. 9. Palatal appliance provided with dental elements to restore the aesthetic function of the child.

\section{Results}

Following periodic inspections, it has been observed that treatment with these devices ensures effective control of the space. These devices have an optimal dimensional stability and do not interfere with the eruption of permanent teeth but also have certain shortcomings such as the possibility of decementation of the orthodontic band or clogging of the loop under the action of masticatory forces.
In some cases where the Nance device has been applied, has been observed in the case of unilateral decementation and clogging of a band in the gingival groove an increase in pressure at the level of the acrylic button, which can cause damage to the palatal mucosa. Also, if there is an increased retention of bacterial plaque between the acrylic button and the mucosa, a local inflammation may occur which requires temporary decementation of the device until the remission of inflammatory phenomena. 
Fixed space maintainers need to be decementated every six months to apply fluorinated gels to the permanent primary molars to achieve caries prophylaxis.

Space maintainers with acrylate can generate an unpleasant odor due to the porosity of the material, if rigorous hygiene of both the oral cavity and these devices is not achieved.

\section{Discussion}

Arikan V et al., 2015, observed that both fixed and removable space maintainers are associated with the presence of a high number of microorganisms in the oral cavity, especially in patients with high bacterial plaque index. Candida has been found frequently in patients with removable space maintainers, while Enterococcus faecalis has been isolated from fixed devices [13].

Various space maintainers such as band and loop, crown and loop and lingual bar are regularly used in space management. Certain disadvantages, such as corrosion and fracture of the device have contributed to the development of more aesthetic and metal-free space maintainers, examples that include composite resin-reinforced fiberglass and the ceramic space maintainer made by CAD-CAM technique [14].
Bishara SE et al. and Agaoglu G et al., highlighted the fact that metal orthodontic devices, used in the oral cavity, corrode in the oral environment releasing both nickel and chromium $[15,16]$. Patients with allergic terrain should be monitored more often because there are studies with patients who have developed metal allergies in the form of gingivitis, burning sensation, gingival hyperplasia, numbness on the tongue edges caused mainly by nickel $[17,18]$.

\section{Conclusions}

Regarding the interceptive therapy with space maintainers, the doctor must establish an individualized work protocol with alternatives of treatment because we are in mixed dentition where the exfoliation process of the primary teeth is active and an unforeseen situation can occur at any time. The space maintainers have an increased efficiency in the interception of the installation of dental anomalies with crowding, without interfering with the physiological processes of growth and development, respectively with the eruption of the permanent successors, being well tolerated by children.

\section{References}

1. Pawar BA. Maintenance of space by innovative threedimensional-printed band and loop space maintainer. J Indian Soc Pedod Prev Dent 2019;37:205-8.

2. Watt E, Ahmad A, Adamji R, Katsimbali A, Ashley P, and Noar J. Space maintainers in the primary and mixed dentition - a clinical guide. British Dental Journal. 2018; 225(4): 293-8.

3. Demars-Fremault C, Pilipili Muhima C. [Furcation lesions in deciduous teeth] Actual Odontostomatol (Paris). 1991 Mar;45(173) 9-23. PMID: 1853749.

4. Setia V, Pandit IK, Srivastava N, Gugnani N, Sekhon HK. Space maintainers in dentistry: Past to present. J Clin Diagn Res 2013;7:2402-5

5. Holan G, Needleman HL. Premature loss of primary anterior teeth due to trauma--potential short- and longterm sequelae. Dent Traumatol. 2014 Apr;30(2):100-6. doi: 10.1111/edt.12081. Epub 2013 Oct 20. PMID: 24138100 .

6. Vinothini V, Sanguida A, Selvabalaji A, Prathima GS, Kavitha M. Functional Band and Loop Space Maintainers in Children. Case Rep Dent. 2019;2019:4312049. Published 2019 Apr 24. doi:10.1155/2019/4312049

7. Sharma S, Goel S. 3D printing and its future in medical world. J Med Res Innov 2019;3:e000141

8. Singh, Pheiroijam Herojit \& Naorem, Herojit \& Chaoba, Thokchom \& Debbarma, Nirmal. (2020). Modern concepts of space maintainers and space regainers: a review article.
9. Soni,H.K.,(2017).Application of CAD -CAM for Fabrication of Metal-Free Band and Loop Space Maintainer, $\quad$ 11(2), ZD14-ZD16. https://www.doi.org/10.7860/JCDR/2017/23459/9246

10. Guo, H., Wang, Y., Zhao, Y. et al. Computer-aided design of polyetheretherketone for application to removable pediatric space maintainers. BMC Oral Health 20, 201 (2020). https://doi.org/10.1186/s12903020-01184-6

11. Ierardo G, Luzzi V, Lesti M, Vozza I, Brugnoletti O, Polimeni A, Bossù M. Peek polymer in orthodontics: A pilot study on children. J Clin Exp Dent. 2017 Oct 1;9(10):e1271-e1275. doi: 10.4317/jced.54010. PMID: 29167720 ; PMCID: PMC5694159.

12. Space Management in Primary and Mixed Dentition, Timişoara 2019, ISBN 978-606-786-144-0.

13. Arikan V, Kizilci E, Ozalp N, Ozcelik B. Effects of Fixed and Removable Space Maintainers on Plaque Accumulation, Periodontal Health, Candidal and Enterococcus Faecalis Carriage. Med Princ Pract. 2015;24(4):311-7. doi: 10.1159/000430787. Epub 2015 Jun 4. PMID: 26044443; PMCID: PMC5588238.

14. Tuloglu N, Bayrak S, Tunc ES. Different clinical applications of bondable reinforcement ribbond in pediatric dentistry. Eur J Dent 2009;3:329-34

15. Bishara SE, Barrett RD, Selim MI. Biodegradation of orthodontic appliances. Part II. Changes in the blood level of nickel. Am J Orthod Dentofacial Orthop. 1993;103(2):115-19. 


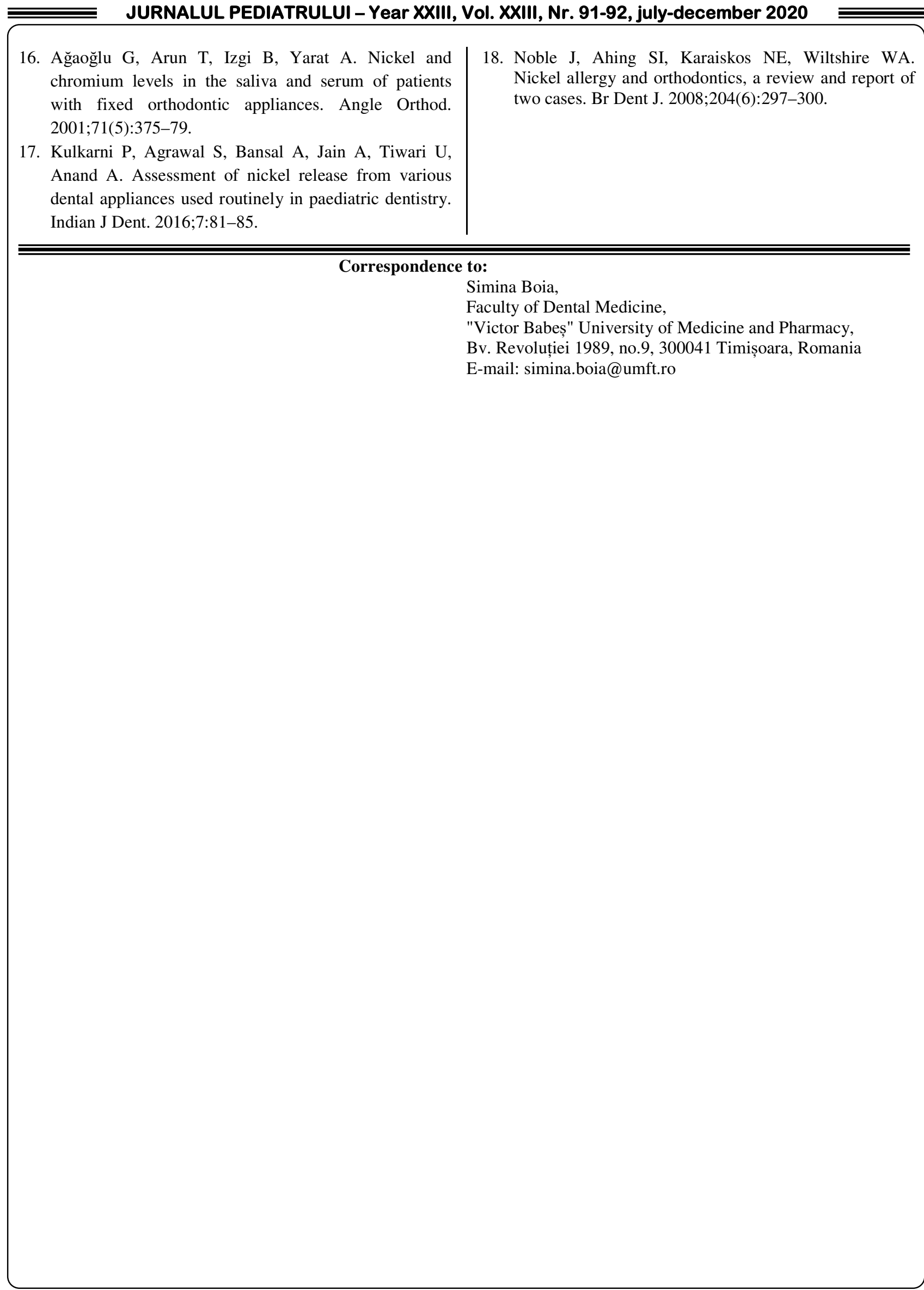

